Article

\title{
On Slight Omega Continuity and Irresoluteness between Generalized Topological Spaces
}

\author{
Samer Al Ghour * and Abeer Al-Nimer \\ Department of Mathematics and Statistics, Jordan University of Science and Technology, Irbid 22110, Jordan; \\ analnimer14@sci.just.edu.jo \\ * Correspondence: algore@just.edu.jo
}

Received: 12 March 2020; Accepted: 4 April 2020; Published: 8 May 2020

\begin{abstract}
We introduce slightly $\omega$ - $\left(\mu_{1}, \mu_{2}\right)$-continuous and slightly $\omega$ - $\left(\mu_{1}, \mu_{2}\right)$-irresolute functions as two new classes of functions on generalized topological spaces. We obtained several properties and characterizations of these functions. Moreover, we deal with preservations of Lindelofness, mild Lindelofness, mildly countable compactness, and connectedness under images or inverse images of a class of functions, which gives symmetric relationships.
\end{abstract}

Keywords: $\omega$-open; slightly $\left(\mu_{1}, \mu_{2}\right)$-continuous; slightly $\omega$ - $\left(\mu_{1}, \mu_{2}\right)$-irresolute; $\left(\mu_{1}, \mu_{2}\right)$-perfect; $\mu$-Lindelof; $\mu$-mildly-Lindelof; $\mu$-mildly-countably-compact

\section{Introduction}

As a stronger notion than accumulation point, a condensation point of a subset $A$ of a topological space $(X, \tau)$ is every point $x$ in $X$ such that every open neighborhood of $x$ uncountably contains many points of $A$. The notions of $\omega$-closed sets and $\omega$-open sets were introduced by Hdeib [1]. Those subsets of a topological space containing their condensation points are called $\omega$-closed sets, and their complements are called $\omega$-open sets. Via $\omega$-open sets, the author in [1] characterized Lindelöf topological spaces; the authors in [2-4] introduced some types of continuity. Moreover, the authors in [5-9] introduced many modifications of both $\omega$-open and $\omega$-closed sets. Recently, the authors in [10-16] obtained several interesting results related to these sets. The authors in [17-19] introduced $\omega$-open sets in fuzzy topological spaces and soft topological spaces. The area of research related to $\omega$-open sets is still a hot topic in the field topology structure and its modifications. As a new mathematical structure, the notion of a generalized topological space (GTS) was initiated by Császár in [20]. The pair $(X, \mu)$, where $\mu$ is a family of subsets of $X$, is called a GTS if $\mu$ contains the empty set and an arbitrary union of members if $\mu$ is a member of $\mu$. For a GTS $(X, \mu)$, the members of $\mu$ are called $\mu$-open sets and their complements are called $\mu$-closed sets, also subsets of $X$ which are $\mu$-open and $\mu$-closed are called $\mu$-clopen. Since Császár defined GTSs, various authors have modified many topological concepts to include the structure of GTSs. In [21], the authors introduced the notion of a slightly $(\mu, \lambda)$-continuous function between GTSs in which they gave some preservation theorems. In a part of this paper, we continue the study of slightly $(\mu, \lambda)$-continuous functions, in particular, we introduce some characterizations and preservation theorems. In [22], the definition of $\omega$-open sets was extended to include GTSs. The work of [22] was continued by [23-25]. In this paper, we extend the study of slightly $\omega$-continuity [26] to include the structure of GTSs, and we introduce and investigate the concept of a slightly $\omega$-irresolute function between GTSs. The study deals with the preservation of Lindelofness, mild Lindelofness, mildly countable compactness, and connectedness under images or inverse images of a class of functions, which gives symmetric relationships. Note that a GTS $(X, \mu)$ contains $\mu$-clopen sets only if $X \in \mu$. Therefore, in this work, for each GTS $(X, \mu)$, we will assume that $X \in \mu$. 
The structure of this paper lies is as follows. In Section 2, we collect some known results regarding slight continuity, we also introduce some new results regarding slight continuity. In Section 3, we introduce and investigate slightly $\omega-(\mu, \sigma)$-continuous functions. In Section 4 , we introduce and investigate slightly $\omega$ - $(\mu, \sigma)$-irresolute functions. In Section 5 , we deal with the preservation of several covering properties under inverse images of a certain class of functions.

Throughout this paper, we follow the notions and terminologies as they appeared in [22]: $\mathbb{Q}$ and $\mathbb{Q}^{c}$ denote the rationals and irrationals, respectively, and $\tau_{u}$ denote the usual topology on $\mathbb{R}$.

\section{Slightly $(\mu, \sigma)$-Continuous Functions}

In 1997, Singal et al. introduced the concept of slight continuity as a generalization of continuity in [27].

The authors in [21] extended slight continuity to include GTSs as follows:

Definition 1. [21] A function $f:\left(X, \mu_{1}\right) \longrightarrow\left(Y, \mu_{2}\right)$ between the GTSs $\left(X, \mu_{1}\right)$ and $\left(Y, \mu_{2}\right)$ is called slightly $\left(\mu_{1}, \mu_{2}\right)$-continuous if $f^{-1}(B)$ is $\mu_{1}$-open for every $\mu_{2}$-clopen subset $B \subseteq Y$.

Theorem 1. [21] For any function $f:\left(X, \mu_{1}\right) \longrightarrow\left(Y, \mu_{2}\right)$ between the GTSs $\left(X, \mu_{1}\right)$ and $\left(Y, \mu_{2}\right)$, the following statements are equivalent:
(a) $f$ is slightly $\left(\mu_{1}, \mu_{2}\right)$-continuous;
(b) For each $\mu_{2}$-clopen set $B \subseteq Y, f^{-1}(B)$ is $\mu_{1}$-closed;
(c) For each $\mu_{2}$-clopen set $B \subseteq Y, f^{-1}(B)$ is $\mu_{1}$-clopen.

Definition 2. Let $X$ be a nonempty set and $\beta$ be a collection of subsets of $X$. Then, the collection of all possible unions of elements of $\beta$ together with $\varnothing$ and $X$ will be denoted by $\beta^{+}$.

Proposition 1. Let $X$ be a nonempty set and $\beta$ be a collection of subsets of $X$. Then, the collection $\beta^{+}$forms a GT on X.

Proof. Obvious.

Each one of the following two bijection functions is not slightly $\left(\beta^{+}, \beta^{+}\right)$-continuous:

Example 1. Let $X=\{1,2,3,4\}$ and $\beta=\{\{1,2\},\{3,4\},\{1,3\},\{2,4\}\}$. Define $f:\left(X, \beta^{+}\right) \longrightarrow\left(X, \beta^{+}\right)$ by

$$
f=\{(1,2),(2,3),(3,4),(4,1)\} .
$$

Let $B=\{1,2\}$. Since $B$ is $\beta^{+}$-clopen but $f^{-1}(B)=\{1,4\}$ is not $\beta^{+}$-open, then $f$ is not slightly $\left(\beta^{+}, \beta^{+}\right)$-continuous.

Example 2. Let $X=\mathbb{R}$ and $\beta=\{[a, b): a, b \in \mathbb{R}$ and $a<b\}$. Define $f:\left(X, \beta^{+}\right) \longrightarrow\left(X, \beta^{+}\right)$by

$$
f(x)=-x^{3} \text {. }
$$

Let $B=[1,8)$. Since $B$ is $\beta^{+}$-clopen but $f^{-1}(B)=(-2,1]$ is not $\beta^{+}$-open, then $f$ is not slightly $\left(\beta^{+}, \beta^{+}\right)$-continuous.

Theorem 2. [21] $\left(\mu_{1}, \mu_{2}\right)$-continuous functions are slightly $\left(\mu_{1}, \mu_{2}\right)$-continuous.

The converse of Theorem 2 need not be true in general as each of the following three examples clarifies: 
Example 3. Let $A=(-\infty, 0), B=(0, \infty), X=\mathbb{R}-\{0\}$ and $\beta=\{A, B,\{-1,1\},\{1,2\}\}$. Define $f:\left(X, \beta^{+}\right) \longrightarrow\left(X, \beta^{+}\right)$by

$$
f(x)=-x .
$$

The $\beta^{+}$-clopen sets of $X$ are $\varnothing, X, A$, and $B$. In addition, $f^{-1}(A)=B$ and $f^{-1}(B)=A$. This shows that $f$ is slightly $\left(\beta^{+}, \beta^{+}\right)$-continuous. On the other hand, since $\{1,2\} \in \beta^{+}$but $f^{-1}(\{1,2\})=\{-1,-2\} \notin \beta^{+}$, then $f$ is not $\left(\beta^{+}, \beta^{+}\right)$-continuous.

Example 4. Let $X=\mathbb{R}^{2}, Y=\mathbb{R}, \beta=\{(a, b) \times(c, d): a, b, c, d \in \mathbb{R}, a<b$ and $c<d\}$, and $\delta=$ $\{(a, b): a, b \in \mathbb{R}$ and $a<b\}$. Define $f:\left(X, \beta^{+}\right) \longrightarrow\left(Y, \delta^{+}\right)$by $f(s, t)=\frac{1}{s}$. Then, $f$ is slightly $\left(\beta^{+}, \delta^{+}\right)$-continuous but not $\left(\beta^{+}, \delta^{+}\right)$-continuous.

Example 5. Let $X=Y=\mathbb{R}, \beta=\{(a, b): a, b \in \mathbb{R}$ and $a<b\}$. Consider the Dirichlet function $f$ : $\left(X, \beta^{+}\right) \longrightarrow\left(Y, \delta^{+}\right)$by $f(x)=0$ if $x$ is rational and $f(x)=1$ if $x$ is irrational. Then, $f$ is slightly $\left(\beta^{+}, \delta^{+}\right)$-continuous but not $\left(\beta^{+}, \delta^{+}\right)$-continuous.

Definition 3. A subset $H$ of a GTS $(X, \mu)$ is called $\delta^{*}$ - $\mu$-open if for each $x \in A$, there is a $\mu$-clopen set $M$ of $(X, \mu)$ such that $x \in M \subseteq H$. Complements of $\delta^{*}$ - $\mu$-open sets are called $\delta^{*}-\mu$-closed.

Proposition 2. Let $H$ be a subset of a GTS $(X, \mu)$. Then,

(a) If $H$ is $\mu$-clopen, then $H$ is $\delta^{*}-\mu$-open;

(b) If $H$ is $\mu$-clopen, then $H$ is $\delta^{*}-\mu$-closed.

Proof. (a) For each $x \in H$, we have $x \in H \subseteq H$ and $H$ is $\mu$-clopen. This shows that $H$ is $\delta^{*}$ - $\mu$-open;

(b) Since $H$ is $\mu$-clopen, then $X-H$ is $\mu$-clopen and by (a), $X-H$ is $\delta^{*}-\mu$-open. Hence, $H$ is $\delta^{*}$ - $\mu$-closed.

The following example shows that the converse of each implication of Proposition 2 need not be true in general:

Example 6. Let $X=\{1,2,3,4\}, A=\{1,2\}, B=\{3,4\}, C=\{1,3\}, D=\{2,4\}$, and $\beta=\{A, B, C, D\}$. Then, $A \cup C$ is $\delta^{*}-\beta^{+}$-open but not $\beta^{+}$-clopen since $X-(A \cup C)=\{4\}$ is not $\beta^{+}$-open. This shows that the converse of Proposition $2(a)$ is not true in general. On the other hand, the set $\{4\}$ is a $\delta^{*}-\beta^{+}$-closed that is not $\beta^{+}$-clopen, which shows that the converse of Proposition $2(b)$ is not true in general.

Theorem 3. For any function $f:\left(X, \mu_{1}\right) \longrightarrow\left(Y, \mu_{2}\right)$ between the GTSs $\left(X, \mu_{1}\right)$ and $\left(Y, \mu_{2}\right)$, the following statements are equivalent:

(a) $f$ is slightly $\left(\mu_{1}, \mu_{2}\right)$-continuous;

(b) $f^{-1}(H)$ is $\mu_{1}$-open for every $\delta^{*}-\mu_{2}$-open set $H \subseteq Y$;

(c) $f^{-1}(H)$ is $\mu_{1}$-open for every $\delta^{*}-\mu_{2}$-closed set $H \subseteq Y$.

Proof. (a) $\Longrightarrow$ (b): Let $H$ be a $\delta^{*}-\mu_{2}$-open subset of $Y$. For each $h \in H$, choose a $\mu_{2}$-clopen subset $H_{h} \subseteq Y$ such that $h \in H_{h} \subseteq H$. By Theorem 1, for each $h \in H, f^{-1}\left(H_{h}\right)$ is $\mu_{1}$-open. We have

$$
f^{-1}(H)=\cup\left\{f^{-1}\left(H_{h}\right) \mid h \in H\right\}
$$

It follows that $f^{-1}(H)$ is $\mu_{1}$-open.

(b) $\Longrightarrow$ (c): Let $H$ be a $\delta^{*}-\mu_{2}$-closed subset of $Y$. Then, $Y-H$ is $\delta^{*}-\mu_{2}$-open and by (b), $X-$ $f^{-1}(H)=f^{-1}(Y-H)$ is $\mu_{1}$-open. Hence, $f^{-1}(H)$ is $\mu_{1}$-closed.

(c) $\Longrightarrow$ (a): Follows from Proposition 2 (b) and part (b) of Theorem 1. 
Definition 4. [28] A GTS $(X, \mu)$ is called $\mu$-connected if we cannot find $H, M \in \mu-\{\varnothing\}$ such that $H \cap M=\varnothing$ and $H \cup M=X$.

Theorem 4. Let $f:\left(X, \mu_{1}\right) \longrightarrow\left(Y, \mu_{2}\right)$ be a function between the GTSs $\left(X, \mu_{1}\right)$ and $\left(Y, \mu_{2}\right)$. If $\left(Y, \mu_{2}\right)$ is $\mu_{2}$-connected, then $f$ is slightly $\left(\mu_{1}, \mu_{2}\right)$-continuous.

Proof. Obvious.

Remark 1. The GTS $\left(X, \beta^{+}\right)$in Example 3 is not $\beta^{+}$-connected as $A, B \in \beta^{+}$are nonempty disjoint sets and $A \cup B=X$. Therefore, the implication in Theorem 4 is not reversible.

Theorem 5. [21] Let $f:\left(X, \mu_{1}\right) \longrightarrow\left(Y, \mu_{2}\right)$ be a surjective $\left(\mu_{1}, \mu_{2}\right)$-continuous function between the GTSs $\left(X, \mu_{1}\right)$ and $\left(Y, \mu_{2}\right)$. If $\left(X, \mu_{1}\right)$ is $\mu_{1}$-connected, then $\left(Y, \mu_{2}\right)$ is $\mu_{2}$-connected.

Definition 5. [21] A GTS $(X, \mu)$ is said to be $\mu$-zero-dimensional if there is $\beta \subseteq \mu$ such that each element $B \in \beta$ is $\mu$-clopen and $\mu=\beta^{+}$.

Theorem 6. [21] Let $f:\left(X, \mu_{1}\right) \longrightarrow\left(Y, \mu_{2}\right)$ be a function between the GTSs $\left(X, \mu_{1}\right)$ and $\left(Y, \mu_{2}\right)$, where $\left(Y, \mu_{2}\right)$ is $\mu_{2}$-zero-dimensional. Then, the following statements are equivalent:

(a) $f$ is $\left(\mu_{1}, \mu_{2}\right)$-continuous;

(b) $f$ is slightly $\left(\mu_{1}, \mu_{2}\right)$-continuous.

Remark 2. Example 4 shows that the condition " $\mu_{2}$-zero-dimensional" on the GTS $\left(Y, \mu_{2}\right)$ cannot be dropped.

Definition 6. [29] Let $(Y, \sigma)$ be a GTS and $H$ a nonempty subset of $Y$. The relative GT of $H$ on $Y$ is the GT $\sigma_{H}=\{H \cap V: V \in \sigma\}$ on $H$. The pair $\left(H, \sigma_{H}\right)$ is called a relative GTS of $(Y, \sigma)$.

Theorem 7. Let $f:\left(X, \mu_{1}\right) \longrightarrow\left(Y, \mu_{2}\right)$ be a slightly $\left(\mu_{1}, \mu_{2}\right)$-continuous function between the GTSs $\left(X, \mu_{1}\right)$ and $\left(Y, \mu_{2}\right)$ and let $H \subseteq X$. Then, the restriction of $f$ to $H,\left.f\right|_{H}:\left(H,\left(\mu_{1}\right)_{H}\right) \longrightarrow\left(Y, \mu_{2}\right)$ is slightly $\left(\left(\mu_{1}\right)_{H}, \mu_{2}\right)$-continuous.

Proof. Let $B$ be a $\mu_{2}$-clopen subset of $Y$. Since $f$ is slightly $\left(\mu_{1}, \mu_{2}\right)$-continuous, $f^{-1}(B)$ is $\mu_{1}$-open. Thus,

$$
\left(\left.f\right|_{H}\right)^{-1}(B)=f^{-1}(B) \cap H \in\left(\mu_{1}\right)_{H} .
$$

Hence, $\left.f\right|_{H}$ is slightly $\left(\left(\mu_{1}\right)_{H}, \mu_{2}\right)$-continuous.

Definition 7. [30] Let $(X, \mu)$ and $(Y, \sigma)$ be two GTSs and let

$$
\beta=\{A \times B: A \in \mu \text { and } B \in \sigma\} .
$$

We call $\beta^{+}$the product of the GTSs $\mu$ and $\sigma$ and denote it by $\mu \sigma$. The GTS $(X \times Y, \mu \sigma)$ is called the product of the GTSs $(X, \mu)$ and $(Y, \sigma)$.

Definition 8. [20] Let $(X, \mu)$ be a GTS and $H$ a subset of $X$. Then,

(a) The $\mu$-closure of $\mathrm{H}$; denoted by $\mathrm{Cl}_{\mu}(H)$; is defined by

$$
C l_{\mu}(H)=\cap\{C: C \text { is } \mu \text {-closed in } X \text { and } H \subseteq C\} ;
$$

(b) The $\mu$-interior of $H$; denoted by $\operatorname{Int}_{\mu}(H)$; is defined by

$$
\operatorname{Int}_{\mu}(H)=\cup\{O: O \text { is } \mu \text {-open in } X \text { and } O \subseteq H\} .
$$


Proposition 3. [30] Let $(X, \mu)$ and $(Y, \sigma)$ be two GTSs, $A \subseteq X$ and $B \subseteq Y$. Then,

(a) $\operatorname{Int}_{\mu \sigma}(A \times B)=\operatorname{Int}_{\mu}(A) \times \operatorname{Int}_{\sigma}(B)$;

(b) $C l_{\mu \sigma}(A \times B)=C l_{\mu}(A) \times C l_{\sigma}(B)$.

Corollary 1. Let $(X, \mu)$ and $(Y, \sigma)$ be two GTSs. If $A$ is $\mu$-clopen and $B$ is $\sigma$-clopen, then $A \times B$ is $\mu \sigma$-clopen.

Definition 9. [31] A GTS $(X, \mu)$ is called $\mu$ - $T_{2}$ if for all $x, y \in X$ with $x \neq y$, there are $\mu$-open sets $H, M$ such that $x \in H, y \in M$ and $H \cap M=\varnothing$.

Definition 10. [21] A GTS $(X, \mu)$ is called $\mu$-clopen- $T_{2}$ if for all $x, y \in X$ with $x \neq y$, there are $\mu$-clopen sets $H, M$ such that $x \in H, y \in M$ and $H \cap M=\varnothing$.

Theorem 8. If $(X, \mu)$ is a $\mu$-clopen- $T_{2} G T S$, where $X$ contains at least two points, then $(X, \mu)$ is not $\mu$-connected.

Proof. Suppose to the contrary that $(X, \mu)$ is $\mu$-connected. Choose $x, y \in X$ with $x \neq y$. Since $(X, \mu)$ is $\mu$-clopen- $T_{2}$, there are $\mu$-clopen sets $A, B$ such that $x \in A, y \in B$, and $A \cap B=\varnothing$. Thus, $A$ and $X-A$ are $\mu$-open nonempty disjoint sets with $A \cup(X-A)=X$.

Theorem 9. $\mu$-clopen- $T_{2}$ GTSs are $\mu-T_{2}$.

Proof. Obvious.

Remark 3. Since $\left(\mathbb{R}, \tau_{u}\right)$ is $\tau_{u}$ - $T_{2}$ and $\tau_{u}$-connected, then by Theorem $8,\left(\mathbb{R}, \tau_{u}\right)$ is not $\tau_{u}$-clopen- $T_{2}$.

Theorem 10. If the GTS $(Y, \sigma)$ is $\sigma$ - $T_{2}$ and $\sigma$-zero-dimensional, then $(Y, \sigma)$ is $\sigma$-clopen- $T_{2}$.

Proof. By $\sigma$-zero-dimensionality of $(Y, \sigma)$, there is $\beta \subseteq \mu$ such that each element $B \in \beta$ is $\sigma$-clopen and $\sigma=\beta^{+}$. To see that $(Y, \sigma)$ is $\sigma$-clopen- $T_{2}$, let $y, z \in Y$ with $y \neq z$. Since $(Y, \sigma)$ is $\sigma$ - $T_{2}$, there are $\sigma$-open sets $U, V$ such that $y \in U, z \in V$ and $U \cap V=\varnothing$. Choose $G, H \in \beta$ such that $y \in G \subseteq U$ and $z \in H \subseteq V$. Then, $G$ and $H$ are $\sigma$-clopen sets such that $y \in G, z \in H$ and $G \cap H=\varnothing$. This shows that $(Y, \sigma)$ is $\sigma$-clopen- $T_{2}$.

In each of the following two examples, the GTS $(X, \mu)$ is $\mu$-clopen- $T_{2}$ but $(X, \mu)$ is not a topological space:

Example 7. Let $X=\mathbb{R}$ and $\beta=\{(-\infty, a): a \in \mathbb{R}\} \cup\{[a, \infty): a \in \mathbb{R}\}$. Then, $\left(X, \beta^{+}\right)$is a $\beta^{+}$-clopen- $T_{2}$ GTS, but $\left(X, \beta^{+}\right)$is not a topological space.

Example 8. Let $X=\{1,2,3,4\}$ and $\beta=\{\{x, y\}: x, y \in X$ and $x \neq y\}$. Then, $\left(X, \beta^{+}\right)$is a $\beta^{+}$-clopen- $T_{2}$ GTS, but $\left(X, \beta^{+}\right)$is not a topological space.

Theorem 11. If $f:(Y, \mu) \longrightarrow(Z, \sigma)$ is a slightly $(\mu, \sigma)$-continuous function, where $(Z, \sigma)$ is $\sigma$-clopen- $T_{2}$, then the graph $G(f)=\{(y, f(y)): y \in Y\}$ of $f$ is $\mu \sigma$-closed.

Proof. Let $(y, z) \in Y \times Z-G(f)$. Then, $f(y) \neq z$. As $(Z, \sigma)$ is $\sigma$-clopen- $T_{2}$, we find $\sigma$-clopen sets $H, M$ such that $f(y) \in H$ and $z \in M$ and $H \cap M=\varnothing$. Since $f$ is slightly $(\mu, \sigma)$-continuous, $f^{-1}(H) \in \mu$. Now, $(y, z) \in f^{-1}(H) \times M \in \mu \sigma$ and $\left(f^{-1}(H) \times M\right) \cap G(f)=\varnothing$. Therefore, $Y \times Z-G(f) \in \mu \sigma$, and hence $G(f)$ is $\mu \sigma$-closed.

Theorem 12. Let $(X, \mu)$ be a GTS and $(Y, \sigma)$ be a $\sigma$-clopen- $T_{2}$ GTS. If $f:(X, \mu) \longrightarrow(Y, \sigma)$ is a slightly $(\mu, \sigma)$-continuous function, then the set $\{(a, b): a, b \in X$ and $f(a)=f(b)\}$ is a $\mu \mu$-closed subset of $X \times X$. 
Proof. Put $H=\{(a, b): a, b \in X$ and $f(a)=f(b)\}$. Let $(a, b) \in(X \times X)-H$. Then, $f(a) \neq f(b)$. Since $(Y, \sigma)$ is $\sigma$-clopen- $T_{2}$, there exist disjoint $\sigma$-clopen sets $C, D$ such that $f(a) \in C$ and $f(b) \in$ $D$. Since $f$ is slightly $(\mu, \sigma)$-continuous, then $f^{-1}(C), f^{-1}(D) \in \mu$. We have $(a, b) \in f^{-1}(C) \times$ $f^{-1}(D) \in \mu \mu$ and $\left(f^{-1}(C) \times f^{-1}(D)\right) \cap H=\varnothing$. This proves that $(X \times X)-H \in \mu \mu$, and hence $H$ is $\mu \mu$-closed.

\section{Slightly $\omega-(\mu, \sigma)$-Continuous Functions}

As a weaker form of continuity between GTSs, the authors in [22] defined $\omega$-continuity between GTSs as follows:

Definition 11. [22] A function $g:\left(Y, \mu_{1}\right) \longrightarrow\left(Z, \mu_{2}\right)$ between the GTSs $\left(Y, \mu_{1}\right)$ and $\left(Z, \mu_{2}\right)$ is called $\omega$ - $\left(\mu_{1}, \mu_{2}\right)$-continuous if for each $\mu_{2}$-open subset $H \subseteq Z, g^{-1}(H)$ is $\omega$ - $\mu_{1}$-open.

Definition 12. A function $g:\left(Y, \mu_{1}\right) \longrightarrow\left(Z, \mu_{2}\right)$ between the GTSs $\left(Y, \mu_{1}\right)$ and $\left(Z, \mu_{2}\right)$ is called slightly $\omega$ - $\left(\mu_{1}, \mu_{2}\right)$-continuous if for each $\mu_{2}$-clopen subset $H \subseteq Z, g^{-1}(H)$ is $\omega$ - $\mu_{1}$-open.

Example 9. Let $\beta$ be as in Example 7. Define $g:\left(\mathbb{R}, \beta^{+}\right) \longrightarrow\left(\mathbb{R}, \beta^{+}\right)$by

$$
g(y)=\left\{\begin{array}{ll}
x & \text { if } y \in \mathbb{Q}^{c} \\
1 & \text { if } y \in \mathbb{Q}
\end{array} .\right.
$$

Then, $g$ is slightly $\omega-\left(\beta^{+}, \beta^{+}\right)$-continuous.

Proof. Note that the set of all $\beta^{+}$-clopen subsets of $\left(\mathbb{R}, \beta^{+}\right)$is $\beta$ together with $\varnothing$ and $\mathbb{R}$. Let $a \in \mathbb{R}$. Then,

$$
g^{-1}((-\infty, a))=\left\{\begin{array}{cc}
(-\infty, a) & \text { if } a>1 \\
(-\infty, a)-\mathbb{Q} & \text { if } a \leq 1
\end{array}\right.
$$

and

$$
g^{-1}([a, \infty))=\left\{\begin{array}{cc}
{[a, \infty)} & \text { if } a \leq 1 \\
{[a, \infty)-\mathbb{Q}} & \text { if } a>1
\end{array} .\right.
$$

Therefore, $g^{-1}(B) \in\left(\beta^{+}\right)_{\omega}$ for all $B \in \beta$. It follows that $g$ is slightly $\omega$ - $\left(\beta^{+}, \beta^{+}\right)$-continuous.

Example 10. Consider the function $f$ as in Example 2. Then,

(a) $\{(a, b]: a, b \in \mathbb{R}$ and and $a<b\} \cap\left(\beta^{+}\right)_{\omega}=\varnothing$;

(b) $f$ is not slightly $\omega-\left(\beta^{+}, \beta^{+}\right)$-continuous.

Proof. (a) Suppose to the contrary that there are $a, b \in \mathbb{R}$ such that $a<b$ and $(a, b] \in\left(\beta^{+}\right)_{\omega}$. Then, there are $U \in \beta^{+}$and a countable subset $C \subseteq \mathbb{R}$ such that $b \in U-C \subseteq(a, b]$. Choose $[s, t)$ such that $b \in[s, t) \subseteq U$. Then, we have $b \in([s, b] \cup(b, t))-C \subseteq(a, b]$, and so $(b, t)$ is countable, which is a contradiction;

(b) $[1,8)$ is $\beta^{+}$-clopen, but by $\left(\right.$a) $f^{-1}([1,8))=(-2,1] \notin\left(\beta^{+}\right)_{\omega}$.

Theorem 13. $\omega-(\mu, \sigma)$-continuity between the $\operatorname{GTSs}(Y, \mu)$ and $(Z, \sigma)$ implies slight $\omega-(\mu, \sigma)$-continuity.

Proof. The proof follows from Proposition 3.4 of [22] and the fact that $\sigma$-clopen sets are $\sigma$-open.

The implication in Theorem 13 is not reversible: 
Example 11. Let $Y=Z=\mathbb{R}$ and $\sigma=\delta=\{\varnothing, \mathbb{R}\} \cup\{\{1,2\},\{2,3\},\{1,2,3\}\}$. Consider $g:(Y, \sigma) \longrightarrow$ $(Z, \delta)$, where

$$
g(y)=\left\{\begin{array}{ll}
1 & \text { if } y \in \mathbb{Q}^{c} \\
3 & \text { if } y \in \mathbb{Q}^{2}
\end{array} .\right.
$$

Since $\{2,3\}$ is a $\delta$-open set but $f^{-1}(\{2,3\})=\mathbb{Q}$ is not $\omega$ - $\sigma$-open, then $g$ is not $\omega$ - $(\sigma, \delta)$-continuous. On the other hand, since the only $\delta$-clopen subsets are $\varnothing$ and $\mathbb{R}$, then $g$ is slightly $\omega$ - $(\sigma, \delta)$-continuous.

Theorem 14. Slight $(\mu, \sigma)$-continuity between the $\operatorname{GTSs}(Y, \mu)$ and $(Z, \sigma)$ implies slight $\omega$ - $(\mu, \sigma)$-continuity.

Proof. Th proof follows from the definitions and the fact that $\mu$-open sets are $\omega$ - $\mu$-open sets.

The following example shows that the converse of Theorem 14 in not true in general:

Example 12. Let $X=\mathbb{R}$ and $\beta=\{\{1\},\{4\}\} \cup\{\{a, b\}: a, b \in \mathbb{R}$ and $a \neq b\}$. Define $f:\left(X, \beta^{+}\right) \longrightarrow$ $\left(X, \beta^{+}\right)$by

$$
f(x)=x^{2}+1
$$

Let $B=\{1\}$. Since $B$ is $\beta^{+}$-clopen but $f^{-1}(B)=\{0\} \notin \beta^{+}$, then $f$ is not slightly $\left(\beta^{+}, \beta^{+}\right)$-continuous. On the other hand, it is easy to check that $\left(\beta^{+}\right)_{\omega}$ is the discrete topology on $X$ and hence $f$ is slightly $\omega-\left(\beta^{+}, \beta^{+}\right)$-continuous.

Theorem 15. If $f:(X, \mu) \longrightarrow(Y, \sigma)$ is a function between the GTSs $(X, \mu)$ and $(Y, \sigma)$, where $(Y, \sigma)$ is $\sigma$-connected, then $f$ is slightly $\omega$ - $(\mu, \sigma)$-continuous.

Proof. Obvious.

Theorem 16. For any function $f:\left(X, \mu_{1}\right) \longrightarrow\left(Y, \mu_{2}\right)$ between the GTSs $\left(X, \mu_{1}\right)$ and $\left(Y, \mu_{2}\right)$, the following statements are equivalent:

(a) $f$ is slightly $\omega$ - $\left(\mu_{1}, \mu_{2}\right)$-continuous;

(b) $f^{-1}(H)$ is $\omega$ - $\mu_{1}$-closed for every $\mu_{2}$-clopen set $B \subseteq Y$;

(c) $f^{-1}(B)$ is $\omega$ - $\mu_{1}$-clopen for every $\mu_{2}$-clopen set $B \subseteq Y$.

Proof. Straightforward.

The converse of Theorem 13 is true if we add the condition ' $\sigma$-zero-dimensional' on the GTS $(Y, \sigma)$ as the following result shows:

Theorem 17. If $f:(X, \mu) \longrightarrow(Y, \sigma)$ is a slightly $\omega$ - $(\mu, \sigma)$-continuous function between the GTSs $(X, \mu)$ and $(Y, \sigma)$, where $(Y, \sigma)$ is $\sigma$-zero-dimensional, then $f$ is $\omega$ - $(\mu, \sigma)$-continuous.

Proof. Let $C \in \sigma-\{\varnothing, Y\}$. Since $(Y, \sigma)$ is $\sigma$-zero-dimensional, there is $\beta \subseteq \sigma$ such that each element $B \in \beta$ is $\sigma$-clopen, and $\sigma=\beta^{+}$. Choose $\beta_{1} \subseteq \beta$ such that $\beta_{1}=C$. By the slight $\omega-(\mu, \sigma)$-continuity of $f$, $f^{-1}(B) \in \mu_{\omega}$ for all $B \in \beta_{1}$. Since

$$
f^{-1}(C)=\cup\left\{f^{-1}(B): B \in \beta_{1}\right\},
$$

then $f^{-1}(C)$ is $\omega$ - $\mu$-open.

As defined in [22], a GTS $(Y, \sigma)$ is said to be $\sigma$-anti-locally countable if the intersection of any two $\sigma$-open sets is either empty or uncountable. 
Proposition 4. Let $(Y, \sigma)$ be $\sigma$-anti-locally countable and $H \subseteq Y$. If $H \in \sigma_{\omega}$, then $C l_{\sigma}(H)=C l_{\sigma_{\omega}}(H)$.

Proof. We may assume $H \neq \varnothing$. It is clear that $C l_{\sigma_{\omega}}(H) \subseteq C l_{\sigma}(H)$. To see that $C l_{\sigma}(H) \subseteq C l_{\sigma_{\omega}}(H)$, let $y \in C l_{\sigma}(H)$ and let $G \in \sigma_{\omega}$ such that $y \in G$. Choose $U \in \sigma$ and a countable set $C \subseteq Y$ such that $y \in U-C \subseteq G$. Since $y \in U \cap C l_{\sigma}(H), U \cap H \neq \varnothing$. Choose $z \in U \cap H$. Since $H \in \sigma_{\omega}$, there exists $W \in \sigma$ and a countable set $D \subseteq Y$ such that $z \in W-D \subseteq H$. Since $z \in U \cap W$ and $(Y, \sigma)$ is $\sigma$-anti-locally countable, then $U \cap W$ is uncountable. Thus, $(U-C) \cap(W-D) \neq \varnothing$ and hence $G \cap H \neq \varnothing$. Therefore, $y \in C l_{\sigma_{\omega}}(G)$.

Definition 13. Let $(Y, \sigma)$ be a GTS and $H \subseteq Y$. Then, $H$ is said to be $\omega$ - $\sigma$-clopen if it is both $\omega$ - $\sigma$-open and $\omega-\sigma$-closed.

Theorem 18. Let $(Y, \sigma)$ be $\sigma$-anti-locally countable and $H \subseteq Y$. Then, $H$ is $\sigma$-clopen if $H$ is $\omega$ - $\sigma$-clopen.

Proof. $\Longrightarrow$ ) Suppose that $H$ is $\sigma$-clopen, then $H$ and $Y-H$ are $\sigma$-open. Thus, $H$ and $Y-H$ are $\omega-\sigma$-open, and hence $H$ is $\omega$ - $\sigma$-clopen.

$\Longleftarrow$ ) Suppose that $H$ is $\omega$ - $\sigma$-clopen. Since $H$ and $Y-H$ are $\omega$ - $\sigma$-open sets, then by Proposition 4

$$
C l_{\sigma}(H)=C l_{\sigma_{\omega}}(H) \text { and } C l_{\sigma}(Y-H)=C l_{\sigma_{\omega}}(Y-H) .
$$

Since $H$ is $\omega$ - $\sigma$-closed, then

$$
C l_{\sigma}(H)=C l_{\sigma_{\omega}}(H)=H \text { and } C l_{\sigma_{\omega}}(Y-H)=Y-H .
$$

Therefore,

$$
C l_{\sigma}(H)=H \text { and } C l_{\sigma}(Y-H)=Y-H
$$

and hence $H$ and $Y-H$ are $\sigma$-closed sets. It follows that $H$ is $\sigma$-clopen.

Theorem 19. If $f:\left(X, \mu_{1}\right) \longrightarrow\left(Y, \mu_{2}\right)$ is a slightly $\omega$ - $\left(\mu_{1}, \mu_{2}\right)$-continuous function between the GTSs $\left(X, \mu_{1}\right)$ and $\left(Y, \mu_{2}\right)$, where $\left(X, \mu_{1}\right)$ is $\mu_{1}$-anti-locally countable, then $f$ is slightly $\left(\mu_{1}, \mu_{2}\right)$-continuous.

Proof. Let $B$ be $\mu_{2}$-clopen. By slight $\omega$ - $\left(\mu_{1}, \mu_{2}\right)$-continuity of $f$ and part (c) of Theorem $16, f^{-1}(B)$ is $\omega$ - $\mu_{1}$-clopen. Since $\left(X, \mu_{1}\right)$ is $\mu_{1}$-anti-locally countable, $f^{-1}(B)$ is $\mu_{1}$-clopen. Therefore, by part (c) of Theorem 1, we get the result.

Theorem 20. For any function $f:\left(X, \mu_{1}\right) \longrightarrow\left(Y, \mu_{2}\right)$ between the GTSs $\left(X, \mu_{1}\right)$ and $\left(Y, \mu_{2}\right)$, the following statements are equivalent:

(a) $f$ is slightly $\omega$ - $\left(\mu_{1}, \mu_{2}\right)$-continuous;

(b) $f^{-1}(B)$ is $\omega$ - $\mu_{1}$-open for every $\delta^{*}-\mu_{2}$-open set $B \subseteq Y$;

(c) $f^{-1}(B)$ is $\omega$ - $\mu_{1}$-closed for every $\delta^{*}-\mu_{2}$-closed set $B \subseteq Y$.

$(a) \Longrightarrow(b)$ : Let $B$ be a $\delta^{*}-\mu_{2}$-open subset of $Y$. For each $b \in B$, choose a $\mu_{1}$-clopen subset $B_{b} \subseteq Y$ such that $b \in B_{b} \subseteq B$. For each $b \in B, f^{-1}\left(B_{b}\right)$ is $\omega$ - $\mu_{1}$-open. We have

$$
f^{-1}(B)=\cup\left\{f^{-1}\left(B_{b}\right) \mid b \in B\right\} .
$$

It follows that $f^{-1}(B)$ is $\omega$ - $\mu_{1}$-open.

$(b) \Longrightarrow(c)$ : Let $B$ be a $\delta^{*}-\mu_{2}$-closed subset of $Y$. Then, $Y-B$ is $\delta^{*}-\mu_{2}$-open and by $(b), X-f^{-1}(B)=$ $f^{-1}(Y-B)$ is $\omega$ - $\mu_{1}$-open. Hence, $f^{-1}(B)$ is $\omega$ - $\mu_{1}$-closed.

$(c) \Longrightarrow(a)$ : Follows from Proposition 2 (b) and part (b) of Theorem 16. 
Definition 14. A GTS $(X, \mu)$ is called $\omega$ - $\mu$-connected if there are no nonempty disjoint $\omega$ - $\mu$-open sets $A, B$ such that $A \cup B=X$.

Theorem 21. If $(X, \mu)$ is an $\omega$ - $\mu$-connected GTS, then $(X, \mu)$ is $\mu$-connected.

Proof. By contraposition. If $(X, \mu)$ is not $\mu$-connected, then there exist $A, B \in \mu-\{\varnothing\}$ such that $A \cup B=X$ and $A \cap B=\varnothing$. Thus, we have $A, B$ as nonempty disjoint $\omega$ - $\mu$-open sets with $A \cup B=X$ which shows $(X, \mu)$ is not $\omega$ - $\mu$-connected.

The implication in the above theorem is not reversible as the following two examples show:

Example 13. Let $\mu=\tau_{u} \cup\left\{\mathbb{Q} \cup U: U \in \tau_{u}\right\}$. Then, the $G T S(\mathbb{R}, \mu)$ is

(a) $\mu$-connected;

(b) not $\omega$ - $\mu$-connected.

Proof. (a) Suppose to the contrary that $(\mathbb{R}, \mu)$ is not $\mu$-connected. Then, there exist nonempty disjoint $\mu$-open sets $A, B$ with $A \cup B=\mathbb{R}$. Since $\left(\mathbb{R}, \tau_{u}\right)$ is $\tau_{u}$-connected, then $A \notin \tau_{u}$ or $B \notin \tau_{u}$. Without loss of generality, we may assume that $A \in \mu-\tau_{u}$. Then, there is $U \in \tau_{u}$ such that $A=\mathbb{Q} \cup U$. So, $B=\mathbb{R}-(\mathbb{Q} \cup U)=\mathbb{Q}^{c} \cap(\mathbb{R}-U) \subseteq \mathbb{Q}^{c}$. However, the only $\mu$-open set contained in $\mathbb{Q}^{c}$ is $\varnothing$, and so $B=\varnothing$ which is a contradiction.

(b) For every $x \in \mathbb{Q},\{x\} \in \mu_{\omega}$; because $\{x\}=\mathbb{Q}-(\mathbb{Q}-\{x\})$ with $\mathbb{Q} \in \mu$ and $\mathbb{Q}-\{x\}$ is a countable subset of $\mathbb{R}$; and $\mathbb{R}-\{x\} \in \tau_{u} \subseteq \mu_{\omega}$. Put $A=\{0\}$ and $B=\mathbb{R}-\{0\}$, then $A, B$ are nonempty disjoint $\mu$-open sets with $A \cup B=\mathbb{R}$. This implies that $(\mathbb{R}, \mu)$ is not $\omega$ - $\mu$-connected.

Example 14. Let $X=\mathbb{Q}$ and $\mu=\{\varnothing, \mathbb{Q}\} \cup\{\{1,2\},\{2,3\},\{1,2,3\}\}$. Then, $(X, \mu)$ is a $\mu$-connected GTS. On the other hand, set $A=\{1,2\}$ and $B=\mathbb{Q}-A$. Then, $A, B$ are nonempty disjoint $\omega$ - $\mu$-open sets with $A \cup B=X$. This implies that $(X, \mu)$ is not $\omega$ - $\mu$-connected.

Theorem 22. Let $(X, \mu)$ be a $\mu$-anti-locally countable GTS. Then, the following are equivalent:

(a) $(X, \mu)$ is $\omega$ - $\mu$-connected;

(b) $(X, \mu)$ is $\mu$-connected.

Proof. (a) $\Longrightarrow$ (b): Follows from Theorem 21.

(b) $\Longrightarrow$ (a): By contraposition. Suppose $(X, \mu)$ is not $\omega$ - $\mu$-connected. Then, there are nonempty disjoint $\omega$ - $\mu$-open sets $A, B$ such that $A \cup B=X$. It is not difficult to see that $A$ and $B$ are $\omega$ - $\mu$-clopen. Thus, by Theorem $18, A$ and $B$ are $\mu$-clopen. Therefore, $(X, \mu)$ is not $\mu$-connected.

Corollary 2. $\left(\mathbb{R}, \tau_{u}\right)$ is $\omega$ - $\tau_{u}$-connected.

Proof. It is not difficult to check that $\left(\mathbb{R}, \tau_{u}\right)$ is $\tau_{u}$-anti-locally countable. Since $\left(\mathbb{R}, \tau_{u}\right)$ is $\tau_{u}$-connected, then by Theorem $22,\left(\mathbb{R}, \tau_{u}\right)$ is $\omega$ - $\tau_{u}$-connected.

The following is a natural question:

Is there an $\omega$ - $\mu$-connected GTS $(X, \mu)$ where $\mu$ is not a topology on $X$ ?

The answer is yes, as the following example shows:

Example 15. Let $\beta=\{(-\infty, a): a \in \mathbb{R}\} \cup\{(a, \infty): a \in \mathbb{R}\}$. Then, $\left(\mathbb{R}, \beta^{+}\right)$is a GTS that is not a topological space. It is not difficult to check that $\left(\mathbb{R}, \beta^{+}\right)$is $\beta^{+}$-anti-locally countable and $\beta^{+}$-connected. Therefore, by Theorem 22, $\left(\mathbb{R}, \beta^{+}\right)$is $\omega$ - $\beta^{+}$-connected.

Theorem 23. Let $f:\left(X, \mu_{1}\right) \longrightarrow\left(Y, \mu_{2}\right)$ be a surjective slightly $\omega$ - $\left(\mu_{1}, \mu_{2}\right)$-continuous function between the GTSs $\left(X, \mu_{1}\right)$ and $\left(Y, \mu_{2}\right)$, where $\left(X, \mu_{1}\right)$ is $\omega$ - $\mu_{1}$-connected, then $\left(Y, \mu_{2}\right)$ is $\mu_{2}$-connected. 
Proof. Suppose to the contrary that $\left(Y, \mu_{2}\right)$ is not $\mu_{2}$-connected. Then, there are $H, M \in \mu_{2}-\{\varnothing\}$ such that $H \cap M=\varnothing$ and $H \cup M=Y$. Since it is clear that $H, M$ are $\mu_{2}$-clopen subsets of $Y$ and $f$ is slightly $\omega$ - $\left(\mu_{1}, \mu_{2}\right)$-continuous, then $f^{-1}(H), f^{-1}(M)$ are $\omega$ - $\mu_{1}$-open sets. Since $f$ is surjective and $H, M$ are nonempty, then $f^{-1}(H), f^{-1}(M)$ are nonempty. In addition,

$$
f^{-1}(H) \cap f^{-1}(M)=f^{-1}(H \cap M)=f^{-1}(\varnothing)=\varnothing,
$$

and,

$$
f^{-1}(H) \cup f^{-1}(M)=f^{-1}(H \cup M)=f^{-1}(Y)=X .
$$

It follows that $\left(X, \mu_{1}\right)$ is not $\omega$ - $\mu_{1}$-connected, which is a contradiction.

\section{Slightly $\omega$ - $(\mu, \sigma)$-Irresolute Functions}

Definition 15. [23] A function $f:(X, \mu) \longrightarrow(Y, \sigma)$ between the GTSs $(X, \mu)$ and $(Y, \sigma)$ is called $\omega$ - $(\mu, \sigma)$-irresolute if for each $\omega$ - $\sigma$-open subset $B$ of $Y$, the inverse image $f^{-1}(B)$ is an $\omega$ - $\mu$-open subset of $X$.

Definition 16. A function $f:(X, \mu) \longrightarrow(Y, \sigma)$ between the $\operatorname{GTSs}(X, \mu)$ and $(Y, \sigma)$ is called slightly $\omega$ - $(\mu, \sigma)$-irresolute if for each $\omega$ - $\sigma$-clopen subset $B$ of $Y$, the inverse image $f^{-1}(B)$ is an $\omega$ - $\mu$-open subset of $X$.

Example 16. Let $\mu=\tau_{u} \cup\left\{\mathbb{N} \cup U: U \in \tau_{u}\right\}$, where $\mathbb{N}$ is the natural numbers. Define $f, g:(\mathbb{R}, \mu) \longrightarrow$ $(\mathbb{R}, \mu)$ by

$$
f(x)=\left\{\begin{array}{cc}
x^{2}+2 & \text { if } x \in \mathbb{N} \\
\sqrt{2} & \text { if } x \in \mathbb{R}-\mathbb{N}
\end{array}\right.
$$

and

$$
g(x)=x^{2}
$$

Then,

(a) $f$ is slightly $\omega-(\mu, \mu)$-irresolute;

(b) $g$ is not slightly $\omega-(\mu, \mu)$-irresolute.

Proof. (a) It is not difficult to check that the set of all $\omega$ - $\mu$-clopen subsets of $(\mathbb{R}, \mu)$ is $\{\mathbb{R}\} \cup$ $\{A: A \subseteq \mathbb{N}$ or $A \subseteq \mathbb{R}-\mathbb{N}\}$. If $A \subseteq \mathbb{N}$, then $f^{-1}(A)=\{x \in \mathbb{R}: f(x) \in A\}=\left\{x \in \mathbb{N}: x^{2}+2 \in A\right\} \subseteq$ $\mathbb{N}$ and so $f^{-1}(A) \in \mu_{\omega}$. If $A \subseteq \mathbb{R}-\mathbb{N}$, then $f^{-1}(A)=A$ when $\sqrt{2} \in A$ and $f^{-1}(A)=\varnothing$ when $\sqrt{2} \notin A$, and so $f^{-1}(A) \in \mu_{\omega}$. It follows that $f$ is slightly $\omega$ - $(\mu, \mu)$-irresolute.

(b) Since $\{2\}$ is $\omega$ - $\mu$-clopen but $g^{-1}(\{2\})=\{-\sqrt{2}, \sqrt{2}\}$ is not $\omega$ - $\mu$-open, then $g$ is not slightly $\omega$ - $(\mu, \mu)$-irresolute.

Theorem 24. Let $f:(X, \mu) \longrightarrow(Y, \sigma)$ be a function between the $\operatorname{GTSS}(X, \mu)$ and $(Y, \sigma)$.

(a) If $f$ is $\omega$ - $(\mu, \sigma)$-irresolute, then $f$ is slightly $\omega-(\mu, \sigma)$-irresolute;

(b) If $f$ is slightly $\omega-(\mu, \sigma)$-irresolute, then $f$ is slightly $\omega$ - $(\mu, \sigma)$-continuous;

(c) If $(Y, \sigma)$ is $\omega$ - $\sigma$-connected, then $f$ is slightly $\omega-(\mu, \sigma)$-irresolute;

(d) If $f$ is $\omega$ - $(\mu, \sigma)$-irresolute, then $f$ is $\omega$ - $(\mu, \sigma)$-continuous.

Proof. (a) Suppose that $f$ is $\omega$ - $(\mu, \sigma)$-irresolute and let $B$ be an $\omega$ - $\sigma$-clopen subset of $Y$. Then, $B$ is $\omega$ - $\sigma$-open. Since $f$ is $\omega$ - $(\mu, \sigma)$-irresolute, then $f^{-1}(B)$ is an $\omega$ - $\mu$-open subset of $X$. Therefore, $f$ is slightly $\omega$ - $(\mu, \sigma)$-irresolute;

(b) Suppose that $f$ is slightly $\omega$ - $(\mu, \sigma)$-irresolute and let $B$ be a $\sigma$-clopen subset of $Y$. Then, $B$ is $\omega$ - $\sigma$-clopen. Since $f$ is slightly $\omega$ - $(\mu, \sigma)$-irresolute, then $f^{-1}(B)$ is an $\omega$ - $\mu$-open subset of $X$. Therefore, $f$ is slightly $\omega-(\mu, \sigma)$-continuous; 
(c) and (d) are obvious.

The converse of Theorem 24 (a) is not true in general as the following example shows:

Example 17. Let $X=Y=\mathbb{R}$ and $\mu=\sigma=\tau_{u}$. Define $f:(X, \mu) \longrightarrow(Y, \sigma)$ by

$$
f(x)=\left\{\begin{array}{cc}
1 & \text { if } x \in \mathbb{Q} \\
-1 & \text { if } x \in \mathbb{Q}^{c}
\end{array} .\right.
$$

By Corollary 2, $(Y, \sigma)$ is $\omega$ - $\sigma$-connected and so $f$ is obviously slightly $\omega$ - $(\mu, \sigma)$-irresolute. On the other hand, since $\mathbb{R}-\{-1\}$ is $\omega$ - $\sigma$-open but $f^{-1}(\mathbb{R}-\{-1\})=\mathbb{Q} \notin \mu_{\omega}$, then $f$ is not $\omega$ - $(\mu, \sigma)$-irresolute.

The converse of Theorem 24 (b) is not true in general as the following example shows:

Example 18. Consider the function $g:(Y, \sigma) \longrightarrow(Z, \delta)$ as in Example 11. Let $\lambda=$ the discrete topology on $\{1,2,3\}, \tau_{c o c}=$ the co-countable topology on $\mathbb{R}$ and $v=$ the family of $\delta$-clopen subsets of $(Z, \delta)$. It is easy to check that $\delta_{\omega}=\tau_{c o c} \cup \lambda$. Thus, $v=\lambda \cup\{\mathbb{R}-C: C \in \lambda\}$. Since $\{3\}$ is $\omega$ - $\delta$-clopen but $g^{-1}(\{3\})=\mathbb{Q}$ is not $\omega$ - $\sigma$-open, then $g$ is not slightly $\omega$ - $(\sigma, \delta)$-irresolute. On the other hand, we proved in Example 11 that $g$ is slightly $\omega-(\sigma, \delta)$-continuous.

Theorem 25. Let $f:(X, \mu) \longrightarrow(Y, \sigma)$ be a function between the GTSs $(X, \mu)$ and $(Y, \sigma)$, and let $g$ : $(Y, \sigma) \longrightarrow(Z, \eta)$ be a function between the GTSs $(Y, \sigma)$ and $(Z, \eta)$.

(a) $g \circ f$ is slightly $\omega-(\mu, \eta)$-irresolute if $f$ is slightly $\omega-(\mu, \sigma)$-irresolute and $g$ is slightly $\omega-(\sigma, \eta)$-irresolute;

(b) $g \circ f$ is slightly $\omega-(\mu, \eta)$-continuous if $f$ is slightly $\omega-(\mu, \sigma)$-irresolute and $g$ is slightly $\omega$ - $(\sigma, \eta)$-continuous;

(c) $g \circ f$ is slightly $\omega$ - $(\mu, \eta)$-continuous if $f$ is slightly $\omega$ - $(\mu, \sigma)$-continuous and $g$ is slightly $(\sigma, \eta)$-continuous.

Proof. (a) Let $C$ be an $\omega-\eta$-clopen subset of $Z$. Since $g$ is slightly $\omega$ - $(\sigma, \eta)$-irresolute, then $g^{-1}(C)$ is $\omega$ - $\sigma$-clopen. Since $f$ is slightly $\omega$ - $(\mu, \sigma)$-irresolute, then $f^{-1}\left(g^{-1}(C)\right)$ is $\omega$ - $\mu$-open subset of $X$. Since

$$
(g \circ f)^{-1}(C)=f^{-1}\left(g^{-1}(C)\right)
$$

then $(g \circ f)^{-1}(C)$ is $\omega$ - $\mu$-open set in X. Hence, $g \circ f$ is slightly $\omega$ - $(\mu, \eta)$-irresolute;

(b) Let $C$ be a $\eta$-clopen subset of $Z$. Since $g$ is slightly $\omega$ - $(\sigma, \eta)$-continuous, then $g^{-1}(C)$ is an $\omega$ - $\sigma$-clopen subset of $Y$. Since $f$ is slightly $\omega$ - $(\mu, \sigma)$-irresolute, then $f^{-1}\left(g^{-1}(C)\right)$ is $\omega$ - $\mu$-open subset of $X$. Since

$$
(g \circ f)^{-1}(C)=f^{-1}\left(g^{-1}(C)\right)
$$

then $(g \circ f)^{-1}(C)$ is $\omega$ - $\mu$-open set in $X$. Hence, $g \circ f$ is slightly $\omega$ - $(\mu, \eta)$-continuous;

(c) Let $C$ be a $\eta$-clopen subset of $Z$. Since $g$ is slightly $(\sigma, \eta)$-continuous, then $g^{-1}(C)$ is $\sigma$-clopen. Since $f$ is slightly $\omega$ - $(\mu, \sigma)$-continuous, then $f^{-1}\left(g^{-1}(C)\right)$ is $\omega$ - $\mu$-open subset of $X$. Since

$$
(g \circ f)^{-1}(C)=f^{-1}\left(g^{-1}(C)\right)
$$

then $(g \circ f)^{-1}(C)$ is $\omega$ - $\mu$-open set in $X$. Hence, $g \circ f$ is slightly $\omega$ - $(\mu, \eta)$-continuous.

Corollary 3. If $f:(X, \mu) \longrightarrow(Y, \sigma)$ is an $\omega$ - $(\mu, \sigma)$-irresolute function between the GTSs $(X, \mu)$ and $(Y, \sigma)$ and $g:(Y, \sigma) \longrightarrow(Z, \eta)$ is a slightly $\omega$ - $(\sigma, \eta)$-continuous function between the GTSs $(Y, \sigma)$ and $(Z, \eta)$, then $g \circ f$ is slightly $\omega-(\mu, \eta)$-continuous function. 
Definition 17. A function $f:(X, \mu) \longrightarrow(Y, \sigma)$ is called $\omega$ - $(\mu, \sigma)$-open function if for every $\omega$ - $\mu$-open subset $H$ of $X, f(H)$ is $\omega$ - $\sigma$-open in $Y$.

Theorem 26. Let $f:(X, \mu) \longrightarrow(Y, \sigma)$ be slightly $\omega$ - $(\mu, \sigma)$-irresolute, $\omega$ - $(\mu, \sigma)$-open, and surjective function between the GTSs $(X, \mu)$ and $(Y, \sigma)$, and let $g:(Y, \sigma) \longrightarrow(Z, \eta)$ be a function between the GTSs $(Y, \sigma)$ and $(Z, \eta)$. Then, the following are equivalent:

(a) $g$ is slightly $\omega-(\sigma, \eta)$-continuous;

(b) $g \circ f$ is slightly $\omega-(\mu, \eta)$-continuous.

proof. (a) $\Longrightarrow(b)$ : Theorem 25 (b).

$(b) \Longrightarrow(a)$ : Let $C$ be a $\eta$-clopen subset of Z. Since $g \circ f$ is slightly $\omega$ - $(\mu, \eta)$-continuous, $(g \circ f)^{-1}(C)$ is $\omega$ - $\mu$-open. Since $f$ is $\omega$ - $(\mu, \sigma)$-open, $f\left((g \circ f)^{-1}(C)\right)$ is $\omega$ - $\sigma$-open. Since $f$ is surjective, then $f\left((g \circ f)^{-1}(C)\right)=f\left(f^{-1}\left(g^{-1}(C)\right)\right)=g^{-1}(C)$. It follows that $g^{-1}(C)$ is $\omega$ - $\sigma$-open.

\section{Covering Properties}

Definition 18. [32] A subset $H$ of a GTS $(Y, \sigma)$ is called $\sigma$-Lindelof relative to $Y$ if every cover of $H$ by $\sigma$-open sets has a countable subcover. A GTS $(Y, \sigma)$ is called $\sigma$-Lindelof if each cover of $Y$ by $\sigma$-open sets has a countable subcover.

Remark 4. A GTS $(Y, \sigma)$ is $\sigma$-Lindelof if $Y$ is $\sigma$-Lindelof relative to $Y$.

Definition 19. A function $g:\left(Y, \mu_{1}\right) \longrightarrow\left(Z, \mu_{2}\right)$ between the GTSs $\left(Y, \mu_{1}\right)$ and $\left(Z, \mu_{2}\right)$ is called $\left(\mu_{1}, \mu_{2}\right)$-closed if $g(C)$ is $\mu_{2}$-closed for each $\mu_{1}$-closed set $C$.

Recall that for a function $g: Y \longrightarrow Z$ and for $z \in Z$, the $\operatorname{set} g^{-1}(z)$ is called a fiber of $g$.

Definition 20. A function $g:\left(Y, \mu_{1}\right) \longrightarrow\left(Z, \mu_{2}\right)$ between the GTSs $\left(Y, \mu_{1}\right)$ and $\left(Z, \mu_{2}\right)$ is called $\left(\mu_{1}, \mu_{2}\right)$-perfect if $g$ is $\left(\mu_{1}, \mu_{2}\right)$-continuous, $\left(\mu_{1}, \mu_{2}\right)$-closed, and its fiber $g^{-1}(z)$ is $\mu_{1}$-compact relative to $Y$ for all $z \in Z$.

Definition 21. A subset $H$ of a GTS $(Y, \sigma)$ is called $\sigma$-mildly-compact relative to $Y$ if every cover of $H$ by $\sigma$-clopen sets has a finite subcover.

Definition 22. [21] A GTS $(Y, \sigma)$ is called $\sigma$-mildly compact if each cover of $Y$ by $\sigma$-clopen sets has a finite subcover.

Remark 5. A GTS $(Y, \sigma)$ is $\sigma$-mildly-compact if $Y$ is $\sigma$-mildly-compact relative to $Y$.

Definition 23. A function $g:\left(Y, \mu_{1}\right) \longrightarrow\left(Z, \mu_{2}\right)$ between the GTSs $\left(Y, \mu_{1}\right)$ and $\left(Z, \mu_{2}\right)$ is called strongly $\left(\mu_{1}, \mu_{2}\right)$-closed if $g(C)$ is $\mu_{2}$-clopen for all $\mu_{1}$-closed set $C$.

Theorem 27. Let $g:\left(Y, \mu_{1}\right) \longrightarrow\left(Z, \mu_{2}\right)$ be strongly $\left(\mu_{1}, \mu_{2}\right)$-closed between the GTSs $\left(Y, \mu_{1}\right)$ and $\left(Z, \mu_{2}\right)$ in which its fibers are $\mu_{1}$-mildly-compact relative to $Y$. If $B$ is $\mu_{2}$-mildly-compact relative to $Z$, then $g^{-1}(B)$ is $\mu_{1}$-mildly-compact relative to $Y$.

Proof. Let $\mathcal{U}=\left\{U_{\alpha}: \alpha \in \Delta\right\}$ be a cover of $g^{-1}(B)$ by $\mu_{1}$-clopen sets. Let $\mathcal{F}$ be the family of all finite subsets of $\Delta$. For all $F \in \mathcal{F}$, set $A_{F}=\left\{U_{\alpha}: \alpha \in F\right\}$. For each $b \in B, g^{-1}(b)$ is $\mu_{1}$-mildly-compact relative to $Y$ and so there exists $F_{b} \in \mathcal{F}$ such that $g^{-1}(b) \subseteq A_{F_{b}}$. Set

$$
\mathcal{H}=\left\{Z-g\left(Y-A_{F}\right): F \in \mathcal{F}\right\} .
$$


Claim. $\mathcal{H}$ is a cover of $B$ by $\mu_{2}$-clopen sets.

Proof of Claim. Let $b \in B$. Then, $g^{-1}(b) \subseteq A_{F_{b}}$ and so $b \in Z-g\left(Y-A_{F_{b}}\right)$ with $\left(Z-g\left(Y-A_{F_{b}}\right)\right) \in$ $\mathcal{H}$. It follows that $\mathcal{H}$ is a cover of $B$. Furthermore, for every $F \in \mathcal{F}, A_{F}$ is $\mu_{1}$-open, $Y-A_{F}$ is $\mu_{1}$-closed, and since $g$ is strongly $\left(\mu_{1}, \mu_{2}\right)$-closed, $g\left(Y-A_{F}\right)$ is $\mu_{2}$-clopen. It follows that $\mathcal{H}$ is a cover of $B$ by $\mu_{2}$-clopen sets.

Since $B$ is $\mu_{2}$-mildly-compact relative to $Z$ and by the Claim, $\mathcal{H}$ is a cover of $B$ by $\mu_{2}$-clopen sets, there are $F_{1}, F_{2}, \ldots, F_{n} \in \mathcal{F}$ such that

$$
B \subseteq\left\{Z-g\left(Y-A_{F_{i}}\right): i=1,2, \ldots, n\right\}
$$

Thus,

$$
\begin{aligned}
g^{-1}(B) & \subseteq\left\{g^{-1}\left(Z-g\left(Y-A_{F_{i}}\right)\right): i=1,2, \ldots, n\right\} \\
& =\left\{\left(Y-g^{-1}\left(g\left(Y-A_{F_{i}}\right)\right)\right): i=1,2, \ldots, n\right\} \\
& \subseteq\left\{\left(Y-\left(Y-A_{F_{i}}\right)\right): i=1,2, \ldots, n\right\} \\
& =\left\{A_{F_{i}}: i=1, \ldots, n\right\} .
\end{aligned}
$$

Put $\Delta_{1}=F_{1} \cup F_{2} \cup \ldots \cup F_{n}$. Then, $\left\{U_{\alpha}: \alpha \in \Delta_{1}\right\}$ is a finite subcover of $\mathcal{U}$. Therefore, $g^{-1}(B)$ is $\mu_{1}$-mildly-compact relative to $Y$.

Corollary 4. Let $g:\left(Y, \mu_{1}\right) \longrightarrow\left(Z, \mu_{2}\right)$ be strongly $\left(\mu_{1}, \mu_{2}\right)$-closed between the GTSs $\left(Y, \mu_{1}\right)$ and $\left(Z, \mu_{2}\right)$ in which its fibers are $\mu_{1}$-mildly-compact relative to $Y$. If $\left(Z, \mu_{2}\right)$ is $\mu_{2}$-mildly-compact, then $\left(Y, \mu_{1}\right)$ is $\mu_{1}$-mildly-compact.

Definition 24. A subset $H$ of a GTS $(Y, \sigma)$ is called $\sigma$-mildly-countably-compact relative to $Y$ if every countable cover of $H$ by $\sigma$-clopen sets has a finite subcover.

Definition 25. A GTS $(Y, \sigma)$ is called $\sigma$-mildly-countably-compact if each countable cover of $Y$ by $\sigma$-clopen sets has a finite subcover.

Remark 6. A GTS $(Y, \sigma)$ is $\sigma$-mildly-countably-compact if $Y$ is $\sigma$-mildly-countably-compact relative to $Y$.

Theorem 28. Let $g:\left(Y, \mu_{1}\right) \longrightarrow\left(Z, \mu_{2}\right)$ be strongly $\left(\mu_{1}, \mu_{2}\right)$-closed between the GTSs $\left(Y, \mu_{1}\right)$ and $\left(Z, \mu_{2}\right)$ in which its fibers are $\mu_{1}$-mildly-countably-compact relative to $Y$. If $B$ is $\mu_{2}$-mildly-countably-compact relative to $\mathrm{Z}$, then $\mathrm{g}^{-1}(B)$ is $\mu_{1}$-mildly-countably-compact relative to $\mathrm{Y}$.

Proof. Let $\mathcal{U}=\left\{U_{\alpha}: \alpha \in \Delta\right\}$ be a countable cover of $g^{-1}(B)$ by $\mu_{1}$-clopen sets. Let $\mathcal{F}$ be the family of all finite subsets of $\Delta$. For all $F \in \mathcal{F}$, set $A_{F}=\left\{U_{\alpha}: \alpha \in F\right\}$. For each $b \in B, g^{-1}(b)$ is $\mu_{1}$-mildly-countably-compact relative to $Y$ and so there exists $F_{b} \in \mathcal{F}$ such that $g^{-1}(b) \subseteq A_{F_{b}}$. Set

$$
\mathcal{H}=\left\{Z-g\left(Y-A_{F}\right): F \in \mathcal{F}\right\}
$$

Claim. $\mathcal{H}$ is a countable cover of $B$ by $\mu_{2}$-clopen sets.

Proof of Claim. Let $b \in B$. Then, $g^{-1}(b) \subseteq A_{F_{b}}$ and so $b \in Z-g\left(Y-A_{F_{b}}\right)$ with $\left(Z-g\left(Y-A_{F_{b}}\right)\right) \in$ $\mathcal{H}$. It follows that $\mathcal{H}$ is a cover of $B$. In addition, for every $F \in \mathcal{F}, A_{F}$ is $\mu_{1}$-open, $Y-A_{F}$ is $\mu_{1}$-closed, and since $g$ is strongly $\left(\mu_{1}, \mu_{2}\right)$-closed, $g\left(Y-A_{F}\right)$ is $\mu_{2}$-clopen. It follows that $\mathcal{H}$ is a countable cover of $B$ by $\mu_{2}$-clopen sets. 
Since $B$ is $\mu_{2}$-mildly-countably-compact relative to $Z$, and by the Claim, $\mathcal{H}$ is a cover of $B$ by $\mu_{2}$-clopen sets, there are $F_{1}, F_{2}, \ldots, F_{n} \in \mathcal{F}$ such that

$$
B \subseteq\left\{Z-g\left(Y-A_{F_{i}}\right): i=1,2, \ldots, n\right\}
$$

Thus,

$$
\begin{aligned}
g^{-1}(B) & \subseteq\left\{g^{-1}\left(Z-g\left(Y-A_{F_{i}}\right)\right): i=1,2, \ldots, n\right\} \\
& =\left\{\left(Y-g^{-1}\left(g\left(Y-A_{F_{i}}\right)\right)\right): i=1,2, \ldots, n\right\} \\
& \subseteq\left\{\left(Y-\left(Y-A_{F_{i}}\right)\right): i=1,2, \ldots, n\right\} \\
& =\left\{A_{F_{i}}: i=1,2, \ldots, n\right\} .
\end{aligned}
$$

Put $\Delta_{1}=F_{1} \cup F_{2} \cup \ldots \cup F_{n}$. Then, $\left\{U_{\alpha}: \alpha \in \Delta_{1}\right\}$ is a finite subcover of $\mathcal{U}$. Therefore, $g^{-1}(B)$ is $\mu_{1}$-mildly-countably-compact relative to $Y$.

Corollary 5. Let $g:\left(Y, \mu_{1}\right) \longrightarrow\left(Z, \mu_{2}\right)$ be strongly $\left(\mu_{1}, \mu_{2}\right)$-closed between the GTSs $\left(Y, \mu_{1}\right)$ and $\left(Z, \mu_{2}\right)$ in which its fibers are $\mu_{1}$-mildly-countably-compact relative to $Y$. If $\left(Z, \mu_{2}\right)$ is $\mu_{2}$-mildly-countably-compact, then $\left(Y, \mu_{1}\right)$ is $\mu_{1}$-mildly-countably-compact.

Theorem 29. Let $g:\left(Y, \mu_{1}\right) \longrightarrow\left(Z, \mu_{2}\right)$ be $\left(\mu_{1}, \mu_{2}\right)$-closed between the GTSs $\left(Y, \mu_{1}\right)$ and $\left(Z, \mu_{2}\right)$ in which its fibers are $\mu_{1}$-Lindelof relative to $Y$. If $B$ is $\mu_{2}$-Lindelof relative to $Z$, then $g^{-1}(B)$ is $\mu_{1}$-Lindelof relative to $Y$.

Proof. Let $\mathcal{U}=\left\{U_{\alpha}: \alpha \in \Delta\right\}$ be a cover $g^{-1}(B)$ by $\mu_{1}$-open sets. Let $\mathcal{F}$ be the family of all countable subsets of $\Delta$. For all $F \in \mathcal{F}$, set $A_{F}=\left\{U_{\alpha}: \alpha \in F\right\}$. For each $b \in B, g^{-1}(b)$ is $\mu_{1}$-Lindelof relative to $Y$ and so there exists $F_{b} \in \mathcal{F}$ such that $g^{-1}(b) \subseteq A_{F_{b}}$. Set

$$
\mathcal{H}=\left\{Z-g\left(Y-A_{F}\right): F \in \mathcal{F}\right\} .
$$

Claim. $\mathcal{H}$ is a cover of $B$ by $\mu_{2}$-open sets.

Proof of Claim. Let $b \in B$. Then, $g^{-1}(b) \subseteq A_{F_{b}}$ and so $b \in Z-g\left(Y-A_{F_{b}}\right)$ with $\left(Z-g\left(Y-A_{F_{b}}\right)\right) \in$ $\mathcal{H}$. It follows that $\mathcal{H}$ is a cover of $B$. Additionally, for every $F \in \mathcal{F}, A_{F}$ is $\mu_{1}$-open, $Y-A_{F}$ is $\mu_{1}$-closed, and since $g$ is $\left(\mu_{1}, \mu_{2}\right)$-closed, $g\left(Y-A_{F}\right)$ is $\mu_{2}$-closed. It follows that $\mathcal{H}$ is a cover of $B$ by $\mu_{2}$-open sets.

Since $B$ is $\mu_{2}$-Lindelof relative to $Z$, and by the Claim, $\mathcal{H}$ is a cover of $B$ by $\mu_{2}$-open sets, there exists a countable subfamily $\mathcal{F}_{1} \subseteq \mathcal{F}$ such that

$$
B \subseteq\left\{Z-g\left(Y-A_{F}\right): F \in \mathcal{F}_{1}\right\}
$$

Thus,

$$
\begin{aligned}
g^{-1}(B) & \subseteq\left\{g^{-1}\left(Z-g\left(Y-A_{F}\right)\right): F \in \mathcal{F}_{1}\right\} \\
& =\left\{\left(Y-g^{-1}\left(g\left(Y-A_{F_{i}}\right)\right)\right): F \in \mathcal{F}_{1}\right\} \\
& \subseteq\left\{\left(Y-\left(Y-A_{F_{i}}\right)\right): F \in \mathcal{F}_{1}\right\} \\
& =\left\{A_{F}: F \in \mathcal{F}_{1}\right\} .
\end{aligned}
$$

Put $\Delta_{1}=\left\{F: F \in \mathcal{F}_{1}\right\}$. Then, $\left\{U_{\alpha}: \alpha \in \Delta_{1}\right\}$ is a countable subcover of $\mathcal{U}$. Therefore, $g^{-1}(B)$ is $\mu_{1}$-Lindelof relative to $Y$. 
Corollary 6. Let $g:\left(Y, \mu_{1}\right) \longrightarrow\left(Z, \mu_{2}\right)$ be $\left(\mu_{1}, \mu_{2}\right)$-perfect between the GTSs $\left(Y, \mu_{1}\right)$ and $\left(Z, \mu_{2}\right)$. If $B$ is $\mu_{2}$-Lindelof relative to $Z$, then $g^{-1}(B)$ is $\mu_{1}$-Lindelof relative to $Y$.

Corollary 7. Let $g:\left(Y, \mu_{1}\right) \longrightarrow\left(Z, \mu_{2}\right)$ be $\left(\mu_{1}, \mu_{2}\right)$-closed between the GTSs $\left(Y, \mu_{1}\right)$ and $\left(Z, \mu_{2}\right)$ in which its fibers are are $\mu_{1}$-Lindelof relative to $Y$. If $\left(Z, \mu_{2}\right)$ is $\mu_{2}$-Lindelof, then $(Y, \mu)$ is $\mu_{1}$-Lindelof.

Corollary 8. Let $g:\left(Y, \mu_{1}\right) \longrightarrow\left(Z, \mu_{2}\right)$ be $\left(\mu_{1}, \mu_{2}\right)$-perfect between the GTSs $\left(Y, \mu_{1}\right)$ and $\left(Z, \mu_{2}\right)$. If $\left(Z, \mu_{2}\right)$ is $\mu_{2}$-Lindelof, then $\left(Y, \mu_{1}\right)$ is $\mu_{1}$-Lindelof.

Definition 26. A subset $H$ of a GTS $(Y, \sigma)$ is called $\sigma$-mildly-Lindelof relative to $Y$ if every cover of $H$ by $\sigma$-clopen sets has a countable subcover.

Definition 27. A GTS $(Y, \sigma)$ is called $\sigma$-mildly-Lindelof if each cover of $Y$ by $\sigma$-clopen sets has a countable subcover.

Remark 7. A GTS $(Y, \sigma)$ is $\sigma$-mildly-Lindelof if $Y$ is $\sigma$-mildly-Lindelof relative to $Y$.

Theorem 30. Let $g:\left(Y, \mu_{1}\right) \longrightarrow\left(Z, \mu_{2}\right)$ be strongly $\left(\mu_{1}, \mu_{2}\right)$-closed between the GTSs $\left(Y, \mu_{1}\right)$ and $\left(Z, \mu_{2}\right)$ in which its fibers are $\mu_{1}$-mildly-Lindelof relative to $Y$. If $B$ is $\mu_{2}$-mildly-Lindelof relative to $Z$, then $g^{-1}(B)$ is $\mu_{1}$-mildly-Lindelof relative to $Y$.

Proof. Let $\mathcal{U}=\left\{U_{\alpha}: \alpha \in \Delta\right\}$ be a cover of $g^{-1}(B)$ by $\mu_{1}$-clopen sets. Let $\mathcal{F}$ be the family of all countable subsets of $\Delta$. For all $F \in \mathcal{F}$, set $A_{F}=\left\{U_{\alpha}: \alpha \in F\right\}$. For each $b \in B, g^{-1}(b)$ is $\mu_{1}$-mildly-Lindelof relative to $Y$ and so there exists $F_{b} \in \mathcal{F}$ such that $g^{-1}(b) \subseteq A_{F_{b}}$. Set

$$
\mathcal{H}=\left\{Z-g\left(Y-A_{F}\right): F \in \mathcal{F}\right\} .
$$

Claim. $\mathcal{H}$ is a cover of $B$ by $\mu_{2}$-clopen sets.

Proof of Claim. Let $b \in B$. Then, $g^{-1}(b) \subseteq A_{F_{b}}$ and so $b \in Z-g\left(Y-A_{F_{b}}\right)$ with $\left(Z-g\left(Y-A_{F_{b}}\right)\right) \in$ $\mathcal{H}$. It follows that $\mathcal{H}$ is a cover of $B$. Furthermore, for every $F \in \mathcal{F}, A_{F}$ is $\mu_{1}$-open, $Y-A_{F}$ is $\mu_{1}$-closed, and since $g$ is strongly $\left(\mu_{1}, \mu_{2}\right)$-closed, $g\left(Y-A_{F}\right)$ is $\mu_{2}$-clopen. It follows that $\mathcal{H}$ is a cover of $B$ by $\mu_{2}$-clopen sets.

Since $B$ is $\mu_{2}$-mildly-Lindelof relative to $Z$, and by the Claim, $\mathcal{H}$ is a cover of $B$ by $\mu_{2}$-clopen sets, there is a countable subfamily $\mathcal{F}_{1} \subseteq \mathcal{F}$ such that

$$
B \subseteq\left\{Z-g\left(Y-A_{F}\right): F \in \mathcal{F}_{1}\right\}
$$

Thus,

$$
\begin{aligned}
g^{-1}(B) & \subseteq\left\{g^{-1}\left(Z-g\left(Y-A_{F}\right)\right): F \in \mathcal{F}_{1}\right\} \\
& =\left\{\left(Y-g^{-1}\left(g\left(Y-A_{F_{i}}\right)\right)\right): F \in \mathcal{F}_{1}\right\} \\
& \subseteq\left\{\left(Y-\left(Y-A_{F_{i}}\right)\right): F \in \mathcal{F}_{1}\right\} \\
& =\left\{A_{F}: F \in \mathcal{F}_{1}\right\} .
\end{aligned}
$$

Put $\Delta_{1}=\left\{F: F \in \mathcal{F}_{1}\right\}$. Then, $\left\{U_{\alpha}: \alpha \in \Delta_{1}\right\}$ is a countable subcover of $\mathcal{U}$. Therefore, $g^{-1}(B)$ is $\mu_{1}$-mildly-Lindelof relative to $Y$. 
Corollary 9. Let $g:\left(Y, \mu_{1}\right) \longrightarrow\left(Z, \mu_{2}\right)$ be strongly $\left(\mu_{1}, \mu_{2}\right)$-closed between the GTSs $\left(Y, \mu_{1}\right)$ and $\left(Z, \mu_{2}\right)$ in which its fibers are $\mu_{1}$-mildly-Lindelof relative to $Y$. If $\left(Z, \mu_{2}\right)$ is $\mu_{2}$-mildly-Lindelof, then $\left(Y, \mu_{1}\right)$ is $\mu_{1}$-mildly-Lindelof.

\section{Conclusions}

In this paper, slight $\omega$ - $\left(\mu_{1}, \mu_{2}\right)$-continuity and slight $\omega$ - $\left(\mu_{1}, \mu_{2}\right)$-irresoluteness are extended to include GTSs. Several results and examples related to these new classes of function are given. Furthermore, mapping theorems regarding to some covering generalized topological properties are introduced. In future studies, the following topics could be considered: (1) the definition of fuzzy $\omega$ - $\left(\mu_{1}, \mu_{2}\right)$-continuity, fuzzy $\omega$ - $\left(\mu_{1}, \mu_{2}\right)$-irresoluteness, fuzzy slight $\omega$ - $\left(\mu_{1}, \mu_{2}\right)$-continuity, and fuzzy slight $\omega$ - $\left(\mu_{1}, \mu_{2}\right)$-irresoluteness in fuzzy generalized topological spaces; (2) the extension of $\theta_{\omega}$-openness and $\theta_{\omega}$-continuity, as it appeared in $[15,16]$, to include GTSs.

Author Contributions: Formal analysis, investigation, and writing-original draft preparation S.A.G. and A.A.-N. All authors have read and agreed to the published version of the manuscript.

Funding: This research received no external funding.

Conflicts of Interest: The authors declare no conflict of interest.

\section{References}

1. Hdeib, H. $\omega$-closed mappings. Rev. Colomb. Matem. 1982, 16, 65-78.

2. Hdeib, H.Z. $\omega$-continuous functions. Dirasat J. 1989, 16, 136-142.

3. Al-Omari, A.; Noorani, M.S.M. Contra- $\omega$-continuous and almost Contra- $\omega$-continuous. Int. J. Math. Math. Sci. 2007, 2007, 40469. [CrossRef]

4. Al-Omari, A.; Noiri, T.; Noorani, M.S.M. Weak and strong forms of $\omega$-continuous functions. Int. J. Math. Math. Sci. 2009, 2009, 174042. [CrossRef]

5. Al-Zoubi, K.Y. On generalized $\omega$-closed sets. Int. J. Math. Math. Sci. 2005, 13, 2011-2021. [CrossRef]

6. Al-Hawary, T.; Al-Omari, A. Between open and $\omega$-open sets. Quest. Answers Gen. Topol. 2006, 24, 67-78.

7. Al-Hawary, T.; Al-Omari, A. Decompositions of continuity. Turk. J. Math. 2006, 30, 187-195.

8. Al-Omari, A.; Noorani, M.S.M. Regular generalized $\omega$-closed sets. Int. J. Math. Math. Sci. 2007, 2007, 16292. [CrossRef]

9. Sarsak, M. A decomposition of $\omega$-almost Lindelöf spaces. Quest. Answers Gen. Topol. 2009, 27, 137-139.

10. Roy, B. On nearly Lindelöf spaces via GT. Proyecciones 2019, 38, 49-57. [CrossRef]

11. Agarwal, P.; Goel, C.K. Delineation of $\omega$-bitopological spaces. Proc. Jangjeon Math. Soc. 2019, 22, 507-516.

12. Al Ghour, S. Theorems on strong paracompactness of product spaces. Math. Notes 2018, 103, 54-58. [CrossRef]

13. Al Ghour, S.; Mansur, K. Between open sets and semi-open sets. Univ. Sci. 2018, 23, 9-20. [CrossRef]

14. Al-Omari, A.; Noiri, T. Characterizations of $z$-Lindelöf spaces. Arch. Math. (Brno) 2017, 53, 93-99. [CrossRef]

15. Al Ghour, S.; Irshedat, B. The topology of $\theta_{\omega}$-open sets. Filomat 2017, 31, 5369-5377.

16. Al Ghour, S.; Irshedat, B. On $\theta_{\omega}$ continuity. Heliyon 2020, 6, e03349. [CrossRef]

17. Al Ghour, S. Three new weaker notions of fuzzy open sets and related covering concepts. Kuwait J. Sci. 2017, 44, 48-57.

18. Al Ghour, S. On several types of continuity and irresoluteness in L-topological spaces. Kuwait J. Sci. 2018, 45, 9-14.

19. Al Ghour, S.; Hamed, W. On two classes of soft sets in soft topological spaces. Symmetry 2020, 12, 265. [CrossRef]

20. Császár, A. GT, generalized continuity. Acta Math. Hungar. 2002, 96, 351-357. [CrossRef]

21. Aljarrah, H.; Noorani, M.M.; Noiri, T. Slightly $(\mu, \lambda)$-continuous functions. Fasc. Math. 2014, 52, 5-13.

22. Al Ghour, S.; Zareer, W. Omega open sets in generalized topological spaces. J. Nonlinear Sci. Appl. 2016, 9, 3010-3017. [CrossRef]

23. Al Ghour, S.; Zareer, W. Some mappings and separation axioms via omega open sets in generalized topological spaces. Nonlinear Stud. 2019, 26, 609-616. 
24. Al-Saadi, H.; Al-Omari, A. Some operators in ideal topological spaces. Mo. J. Math. Sci. 2018, 30, 59-71. [CrossRef]

25. Yildirim, E.D. $\omega$-continuity on generalized neighbourhood systems. Commun. Fac. Sci. Univ. Ank. Ser. A1 Math. Stat. 2019, 68, 1974-1982.

26. Noiri, T.; Al-Omari, A.; Noorani, M.M. Slightly $\omega$-continuous functions. Fasc. Math. 2009, 41, 97-106.

27. Singal, A.R.; Jain, R.C. Slightly continuous mappings. J. Indian Math. Soc. (N.S.) 1997, 64, 195-203.

28. Shen, R.-X. A note on generalized connectedness. Acta Math. Hungar. 2009, 122, 231-235. [CrossRef]

29. Al Ghour, S.; Al-Omari A.; Noiri, T. On homogeneity and homogeneity components in generalized topological spaces. Filomat 2013, 27, 1097-1105.

30. Császár, A. Product of generalized topologies. Acta Math. Hungar. 2009, 123, 127-132. [CrossRef]

31. Sarsak, M.S. New separation axioms in generalized topological spaces. Acta Math. Hungar. 2011, 132, $244-252$. [CrossRef]

32. Thomas, J.; John, S.J. $\mu$-compactness in generalized topological spaces. J. Adv. Stud. Topol. 2012, 3, 18-22. [CrossRef]

(c) 2020 by the authors. Licensee MDPI, Basel, Switzerland. This article is an open access article distributed under the terms and conditions of the Creative Commons Attribution (CC BY) license (http:// creativecommons.org/licenses/by/4.0/). 